\title{
Die Erinnerungen des Leonard S.
}

\section{Zum performativen Zweifel in Christopher Nolans Memento}

Hartmut von Sass

für Hannah F.

\author{
I.
}

Ärgerlich, traurig oder beunruhigt $\mathrm{zu}$ werden und nicht $\mathrm{zu}$ wissen weshalb, kann unangenehm sein. Sich nicht erinnern können, jemanden vergessen $\mathrm{zu}$ wollen, mag tragisch erscheinen. Sich vieles merken zu müssen ohne inneren Kompass für neue Evidenzen, ist existentiell verheerend. Literatur und Kino unternehmen immer wieder gefährliche Expeditionen in die Randgebiete des Bewusstseins, die sich im Verlauf der Inszenierung als meist gar nicht so marginal erweisen, sondern zuweilen als Spurenelemente des allzu Gewöhnlichen präsentiert werden; denn Vergessen ist ebenso menschlich wie ihr subtileres Geschwister, die Verdrängung; und Lücken, Sprünge oder Risse im Erinnern bleiben ebenso vertraut wie die Weisen ihrer Frakturen, die in Traum und Halluzination oder gar als irreversible Erosion bei Demenz, Alzheimer und Senilität um sich greifen.

Doch das belletristisch-cineastische Interesse am Geistigen samt all seiner zunehmenden Bedrohungen sollte nicht demographisch imVerweis auf die rapide alternde Gesellschaft gerechtfertigt werden. Vornehmlich handelt es sich um eine spätmoderne Faszination am Morbiden, um eine variantenreich deklinierende Fokussierung $e x$ negativo als schärfender Spiegel des angeblich Gesunden. Jetzt geht es nicht mehr um klare und distinkte Ideen einer denkenden Substanz, sondern um ihre Fehltritte, ihre List gegen sich selbst, ihre weissen Flecken in der denkend orientierenden Topographie. Nach der religiösen Verinnerlichung im Zuge der Reformation, der Promovierung der res cogitationes im frühneuzeitlichen Diskurs und schliesslich all den revolutionären Abtragungen eines allzu ungebrochenen Vernunftglaubens folgt nun der phänomenologische Widerpart einer wahrhaften Dialektik der Aufklärung: die Konzentration aufs Pathologische. 
Das muss nicht als unzeitige Behauptung eines neuen Trends der Kunst verstanden werden - ganz abgesehen davon, dass diese recht schlichte These ohnehin verspätet käme. Und dennoch ist das quasi Kierkegaardsche Manöver, sich dem Bewusstsein, dem >Geist` und der Erinnerung nicht länger über ihre standardisierte Architektonik zu nähern, sondern gerade über deren facettenreichen Zusammenbruch ein Zugang, der erst allmählich an Prominenz gewinnen konnte. Während etwa Martin Suters kürzlich verfilmter Roman Small World (1997) als erster Teil einer "neurologischen Trilogie« von der (dann überaus prekären) Lichtung der Erinnerung an früheste Kindheit gerade in Umkehrung zum sonstigen Gedächtnisverlust der Hauptfigur handelt, ist Leonard Shelby als Mementos Anti-Held (gespielt von Guy Pearce) ganz anders justiert. In Christopher Nolans zweitem Film als dessen Durchbruch zum Westküsten-Pazifik haben wir es mit einem jungen Wittwer zu tun, der seine Frau (angeblich?) durch einen brutalen Überfall verloren hat. Sie wurde vergewaltigt und ermordet, während er einen der Täter erschiessen konnte, um vom anderen überwältigt zu werden. Dabei verlor er die Fähigkeit, neue Ereignisse länger und nachhaltig abspeichern zu können. Der junge Shelby leidet seitdem an sog. »anterograder Amnesie«: Sein Gedächtnis bis vor dem Überfall funktioniert wie gewohnt, Geschehnisse danach kann es jedoch nicht mehr erinnernd festhalten. Ausgerechnet dieser Shelby, der »diesen Zustand« hat, wie er es nennt, will sich für den Mord an seiner Frau und die Zerstörung seines Sinns fürs Vergangene rächen. Wie aber aufklären, ohne sich die Indizien merken zu können? Wie Rache empfinden, wenn man sogleich vergisst, sie vollzogen zu haben? Memento erzählt Shelbys Suche nach dem zweiten Täter angesichts seines seltsamen "Zustands".

II.

Die Schlichtheit der Inszenierung, ja der Story insgesamt steht in bewusstem Kontrast zur unkonventionellen Erzähltechnik des Films. In der Tradition des späten film noir schneidet Nolan einerseits alles Extravagante heraus: durchgängige Reduktion aufs Wesentliche ohne ornamentale Zugaben, keine Besonderheiten der Schauplätze bis hin zum Rudimentär-Anonymen, sehr überschaubares Figurenkabinett ohne Nachzeichnung irgendeiner inneren Dramatik, Verzicht aufs Tragische samt einer Spannung, die sich der Geschichte selbst verdanken würde. Andererseits ist der Film sehr wohl spannend, aber nicht 
durch das, was erzählt wird, sondern durch den narrativen Modus selbst: Die Szenen sind anachron angeordnet; die erste Sequenz ist das eigentliche Ende der Handlung, deren Anfang am Ende gezeigt wird. Erzählebene und erzählte Ebene nähern sich im Verlauf an, um sich einmal zu kreuzen und darauf wieder getrennte Wege zu gehen. Durchbrochen ist der farbige Handlungsstrang durch lineare Schwarzweiss-Sequenzen, in denen Shelby beim Telefonieren mit einem Unbekannten zu sehen ist, dem er von einem ehemaligen Klienten erzählt, der an "anterograder Amnesie» litt. Aus der Verquickung der beiden vordergründig simplen Handlungslinien und deren chiastischen Zeitpfeil bezieht der Film seine originelle Komplexität.

Worum geht's genau? (Eine gute Inhaltsangabe findet sich bei wikipedia, deren Artikel ich hier etwas verändere und ergänze.) Der Film beginnt mit einer rückwärts ablaufenden Farbszene, in der Leonard einen gewissen Teddy erschiesst, um ein Foto von der Leiche zu machen. Der Film springt dann zum chronologischen Beginn der Geschichte: eine Schwarzweiss-Szene, in der Leonard in einem Motel aufwacht und ein Telefongespräch mit dem Unbekannten führt. Leonard erzählt ihm die Geschichte von Sammy Jankis, der durch besagte Amnesie keine neuen Erinnerungen mehr speichern konnte. Zu dieser Zeit war Leonard Ermittler in Sachen Versicherungsbetrug. Seine Aufgabe war herauszufinden, ob Sammys Zustand einer physischen Verletzung entsprang und somit von seiner Versicherung abgedeckt war. Nach mehreren Tests folgerte Leonard, dass Sammys Zustand psychologischer Art war, woraufhin der Versicherungsanspruch abgewiesen wurde. Sammys Frau, Diabetikerin und überzeugt davon, dass Sammy durch einen ausreichend starken Anlass aus seinem Zustand herauskatapultiert werden könnte, brachte ihn in einem letzten Test dazu, ihr mehrfach eine Insulinspritze zu verabreichen. Sie starb, nachdem er ihr durch die Amnesie unwissentlich eine tödliche Überdosis verabreicht hatte. Sammy wurde in eine psychiatrische Klinik eingewiesen.

Sprung zurück in die farbigen Sequenzen: Leonard entwickelt nun ein System aus Polaroids, Notizen und Tätowierungen wichtiger Fakten, um sein fehlendes Kurzzeitgedächtnis gleichsam durch Externalisierung des Speichers auszugleichen. Einer der wenigen Hinweise auf die Identität des zweiten Einbrechers ist ein Tattoo mit dem Namen »John G. «. Der mysteriöse Anrufer erzählt Leonard, dass der Mörder, ein Drogendealer, sich in einem verlassenen Gebäude aufhält. Leonard fährt dorthin und tötet einen Mann namens Jimmy 
Grants. An dieser Stelle des Films wird die Schwarzweiss-Szene zur letzten (aber chronologisch ersten) Farbszene.

Einige Minuten später kommt Teddy an und Leonard findet heraus, dass er benutzt wurde. Grants war zwar Drogendealer, hatte aber nichts mit dem Mord an Leonards Frau zu tun. Im eigentlichen Höhepunkt des Films (chronologisch fast am Anfang, erzählt aber gegen Filmende) wird Leonard von Teddy, der als Polizist für den Fall verantwortlich gewesen war, eröffnet, dass seine Frau den Angriff überlebte und erst später an einer Überdosis Insulin - verabreicht durch Leonard - starb. Laut Teddy war Sammy Jankis ein unverheirateter Betrüger. Allerdings bietet Teddy im selben Gespräch eine erweiterteVersion der Geschehnisse, wonach Leonard den zweiten Mörder durch Teddys Hilfe gefunden und getötet hat. Die Genugtuung der Rache habe jedoch nicht angehalten, sodass ein weiterer John G. (also Jimmy Grants) sterben musste - weitere Tote in Zukunft nicht ausgeschlossen. Teddy behauptet, Leonard aus Mitleid geholfen zu haben, den wahren John G. zu finden - bereits mehr als ein Jahr sei das her, was Leonard natürlich längst unbekannt ist. Nun gibt Teddy aber auch zu, Leonard dahingehend manipuliert zu haben, Jimmy für eine Menge Geld, das dieser für einen Deal im Auto hatte, zu töten.

Bevor Leonard Teddys Enthüllungen vergessen kann, richtet er sich darauf ein, Teddy umzubringen. Er notiert dessen Nummernschild und schreibt eine Notiz, sich diese Information tätowieren zu lassen. Leonard nimmt Jimmys Kleidung und Auto, lässt Teddy zurück und fährt zu einem Tattooshop. Dort findet Leonard eine Notiz von Jimmys Freundin, Natalie, in seiner Tasche. Da er bereits vergessen hat, dass er die Kleidung von Jimmy trägt, denkt er, die Notiz sei für ihn bestimmt. Er fährt zu der Bar, in der Natalie arbeitet, trifft sich mit ihr und erzählt ihr von seinem »Zustand «. Natalie bietet ihm ihre Hilfe an, betrügt ihn aber später und führt ihn dazu, einen Mann namens Dodd zu bedrohen, der Natalie wegen des Geldes von Jimmys Drogengeschäften belästigt hat. Nach einigen Schwierigkeiten zwingt Leonard Dodd zum Verlassen der Stadt. Als Natalie erfährt, dass Dodd fort ist, lässt sie für Leonard das Nummernschild von seiner Tätowierung prüfen. Sie gibt ihm eine Führerscheinkopie des Wagenbesitzers, und Leonard vergleicht die Kopie mit seinem Foto von Teddy, dessen wahrer Name John Edward Gammell ist - (ein) John G. Leonard schliesst daraus, dass Teddy der Vergewaltiger und Mörder seiner Frau ist. Er bringt Teddy zu dem verlassenen Gebäude, in dem er bereits Jimmy Grants ermordet hat, und erschiesst ihn wie in der ersten Szene gezeigt. Leonard macht ein Foto des Toten. 
III.

Wem glauben? Woran (nicht) zweifeln? Shelby ist sich dieses Problems überaus bewusst, was ihn dazu führt, das bereits skizzierte System externer Erinnerung aufzubauen. Wenn er sich selbst nicht erinnern kann, müsse - so der Held - ein anderer Träger der Information etabliert werden. Seine relevanten Gehirnareale werden mittels einer separaten Festplatte substituiert in Form von Fotos samt Notizen zu ihnen und Tätowierungen, die die essentiellen "Fakten" enthalten. Doch daraus erstehen nun eine ganze Reihe von Problemen, von denen zwei besonders hervorstechen: zum einen, dass Informationen nicht unkontaminiert bleiben, zumal sie stets der potentiellen Interpretierbarkeit und der schon längst geschehenen Deutung unterliegen; zum anderen, dass (wie in allen dualistischen Szenarien) eine Brücke zwischen Speicher und Person, die auf das Gespeicherte zurückgreifen will, vorhanden sein muss. Zum ersten Problem kommen wir gleich; dem zweiten Komplex widmen wir uns jetzt.

Shelbys Amnesie lässt Konditionierung zu. Durch Wiederholung von Abläufen und fixierten Routinen kann er dem Nichts seiner zusammenfallenden Erinnerung Entscheidendes entreissen. Dies ist wesentlich für das Funktionieren der Story, denn erst diese Konditionierung, die im Falle von Sammy Jankis offenbar nicht greifen konnte, ermöglicht es Leonard, sich daran zu erinnern, dass er externe Erinnerungen schaffen muss, wie er dies am besten bewerkstelligt und dass er sie überhaupt besitzt. Auf diese Weise entsteht die notwendige Brücke zwischen Person und Speicher. Was noch bei Descartes die Zirbeldrüse als Schaltstelle zwischen Körper und Geist sein sollte, übernimmt in Nolans Skript eine selbst terminierte Abrichtung.

Shelby weiss, dass ihm keine Alternative bleibt. Er könnte sonst sein Leben "nicht über die Bühne bringen", wie er einmal angesichts der drohenden Unmöglichkeit, es überhaupt zu führen, sagt. Als "organisiert" und "zielgerichtet" beschreibt er sich, ganz in der Intentionalität aufgehend - auch im existentiellen Sinn, denn ohne die Rache(phantasie) hat, so scheint es, sein Leben kaum mehr einen Sinn. Und so zückt Shelby stets seine Polaroid-Kamera, um sein Hotel, seinen Jaguar oder seine beiden zwielichtigen Bekannten, Teddy und Natalie, zu fotografieren, um nicht gänzlich zu vergessen, wo er wohnt, womit er sich bewegt und wen er eigentlich kennen sollte (was in seiner Absurdität für einige lustige, auch [Selbst]Ironie verratende Szenen sorgen wird). 
Was sich in dieser Strategie abzeichnet, ist das Dilemma zwischen Wiederholungszwang und Neuigkeitsverarbeitung. Shelby ist nur fähig zu existieren, wenn er in seiner Routine bleibt, die bedroht wird durch einströmende Neuigkeiten, welche wiederum erforderlich sind, um den Fall zu lösen, ohne den er als existentielle Sinnstiftung nicht leben kann. Eben weil Shelby dies einigermassen beherrscht, ist er ein Held; aber weil seine Existenz eben von derartigen Begrenzungen diktiert wird, ist er ein Anti-Held, ein Vertreter des "postheroischen Zeitalters».

\section{IV.}

Shelby wendet sich auf recht ausgeklügelte Weise dem Naheliegenden zu: dem Schaffen von archimedischen Punkten in der Indiziensammlung. Er nennt sie »Fakten«, die nummeriert auf seinem Körper als Tattoos erscheinen oder den Kommentar am Polaroid-Rand bilden. In gewisser Hinsicht macht Shelby aus seiner Not eine Tugend: Hätte er Erinnerungen, wie sie ohne seinen "Zustand " möglich wären, unterlägen diese dem Vergessen, dem gewollten Verdrehen, dem gewählten Unterlassen, dem zersetzenden Zweifel. Shelby hingegen ist insofern Rationalist cartesianischer Schule, als er tatsächlich an letzte Evidenzen glaubt: Nicht Interpretationen zählen, sondern Tatsachen - so werde ermittelt, wie er seinem Begleiter Teddy vorträgt:

"Memory can change the shape of a room; it can change the color of a car. And memories can be distorted. They're just an interpretation, they're not a record, and they're irrelevant if you have the facts."

Gerade durch Shelbys Form der Amnesie ist folglich eine der häufigsten Quellen der Konfusion exkludiert: die Interpretierbarkeit - so jedenfalls die dann enttäuschte Hoffnung. Durch "Fakten", die dem Zweifel entzogen seien, könne ausgeschlossen werden, Formen, Farben und alle weiteren "sekundären Qualitäten" erinnernd zu manipulieren. Es gibt also etwas, das "properly basic« sei. Shelbys Aufgabe ist es, eben an diesen sicheren Ort zu gelangen.

Doch seine Sicht erhält erste Risse, wenn er später in einer der seltenen Szenen, die die Verzweiflung an seiner Erinnerungslosigkeit zumindest anspielt, das menschliche In-der-Welt-Sein in einem ganz anderen Jargon beschreibt: 
Shelby: "There are things you know for sure."

Natalie: »Such as?»

Shelby: "I know what that's going to sound like when I knock on it. I know what that's going to feel like when I pick it up. See? Certainties. It's the kind of memory that you take for granted."

Obwohl Shelby keine philosophischen Ambitionen verrät, klingt er hier fast wie der ganz späte Wittgenstein, der auf seinem Sterbebett Über Gewißheit schrieb. `Dinge für selbstverständlich nehmen<, sich vor einem unbefragten Hintergrund an Selbstverständlichkeiten bewegen, eben: `Gewissheiten haben (obgleich Wittgenstein gerade nicht sagen würde, man 'wisse das Gewisse). Worin nun Shelbys problematische Schlussfolgerung besteht, ist der Übergang von solchen Gewissheiten, die zu bezweifeln vollkommen unsinnig wäre, zur Ansicht, seine kommentierten Fotos und lakonischen Tattoos seien interpretationsunbedürftige "Fakten", denen dieselbe Unerschütterlichkeit zuzugestehen sei, wie es Wittgensteinianer im Falle der Gewissheiten tun.

Memento lebt nun davon, dass Shelbys zunächst erstaunlich gut funktionierendes System vermeintlicher Gewissheiten sukzessive ins Wanken gerät. Also noch einmal die Frage: Wem glauben? Woran (nicht) zweifeln? Vielleicht aber eher: Kann man überhaupt noch jemandem glauben, auch sich selbst? Lässt sich überhaupt noch etwas dem Zweifel entnehmen (im doppelten Sinn)? Es sieht jedenfalls schlecht aus:

Zunächst wird die Brüchigkeit der Fakten immer sichtbarer, vor allem dadurch, dass ihre Genese immer tiefer untergraben wird. Wie angedeutet: Shelby als Ermittler a.D. in Sachen Versicherungsbetrug sieht das ganz anders. Er repräsentiert eine geradezu anti-nietzscheanische Position, indem er ganz dezidiert die Komplementarität zwischen Tatsachen und Interpretationen behauptet. Gerade diesen gegenseitigen Ausschluss gibt er als Grunddevise des Detektivischen aus: Richte Dich nach Fakten und schliesse das deutende Moment strikt aus! Nietzsche nennt Vertreter dieses Schlages Metaphysiker lächelnd in Spott und Mitleid; auch Memento wird Nietzsche Recht geben. Drei kleine Beispiele:

Leonard gerät einmal nach einem Streit mit Natalie in die Situation, sich unbedingt einprägen zu müssen, dass sie ihn benutzt. Sie selbst hatte es ihm in dem Wissen darum, dass er es sofort vergessen wird, zu ihrem Vergnügen gesagt. Nachdem sie alles Schreibzeug beiseite geschafft und ihre diabolische Nachricht dem bald wieder ahnungs- 
losen Opfer hat zukommen lassen, verlässt sie das Haus, nicht aber um wirklich wegzufahren, sondern um im Auto zu warten, wie die Zeit verstreicht, in der sich Shelby händeringend um einen Stift bemüht - »keep concentrated «. Er findet ihn nicht, sie tritt herein, vorgebend, gerade nach Hause zu kommen, er kann sich an nichts erinnern.

Shelby macht Fehler, wobei in Memento der Bezug zwischen Realem und davon Divergentem zusehends verschwimmt, wodurch sich zugleich das, was als >Fehler firmiert, immer stärker dynamisiert. Nach Teddys Enthüllungen zu Leonards angeblich wahrer Vergangenheit notiert er sich dessen Nummernschild. Die Plakette wird später Teddy alias John Edward Gammell - John G. - überführen, spielt also eine kleine, aber seinerseits ambivalente Rolle. Im Kennzeichen kommt einmal $>$ I vor, das Leonard als >1< liest. John G. könnte man wohl auch trotz dieses Lapsus überführen, doch interessanterweise wandelt sich im Filmverlauf die Nummer tatsächlich in die fehlerhafte Version. Shelby hatte durchaus Recht: "Memory can change the shape of a room; it can change the color of a car. And memories can be distorted. They're just an interpretation, they're not a record." Erst die darauf folgende Wendung führt das fragwürdige Moment in seine Sicht hinein: Interpretationen sind eben nicht "irrelevant if you have the facts«. Dieser Satz ist seinerseits kein Fakt, sondern eine Interpretation.

Shelby vergisst nicht nur rasant und er manipuliert nicht nur sich selbst und seine Umgebung, sondern er unterliegt der dauernden Selektion der Fakten, teils aus eigener Wahl, teils als Resultat der Umstände. Ihm scheint das manches Mal selbst als Problem, bisweilen sogar als Entlastung bewusst zu sein; so fragt er sich, ob er zulassen dürfe, dass er vergesse, was gerade gesagt worden sei. Der Suche nach einem Mittel zur Konservierung der "Fakten", sei es ein Stift, sei es die Kamera, geht folglich eine halb durchsichtige Wahl voraus, sich überhaupt auf die Suche zu begeben. Selbst wenn es also - contra Nietzsche - Fakten vor jeder Interpretation gäbe, stellte sich demnach die Schwierigkeit ein, mit ihnen in Verwahrung oder deren Unterlassung umzugehen. Damit verdoppelt sich die Kritik an der Reinheit des Faktischen: Sie sind nicht nur gebunden an einen deutenden Zugang $z u$ ihnen, sondern ebenso an einen praktischen Umgang mit ihnen. 


\section{V.}

Soweit die drei Beispiele. Die Erosion des Faktischen hat aber noch eine weitere Ebene. Nicht nur die "Fakten" selbst geraten ins Zwielicht, sondern auch der, für den sie "Fakten" sind: Leonard Shelby. Seine Ausgangsposition ist für Ermittlungen nicht die günstigste. Damit ist nicht primär sein "Zustand " gemeint, sondern seine Involviertheit in den Fall, seine existentielle Verwobenheit mit dem Überfall (als Opfer), aber auch mit der nun laufenden Suche (als Sinnstiftung). Dadurch hält er sich selbst nicht mehr ans rein Faktische, sondern sein puristisches Gerichtetsein auf NichtInterpretiertes wird nun garniert mit einem "will to believe«, der - ganz im Sinne des pragmatistischen Erfinders - durch den Glaube an $x$, dieses $x$ wahrscheinlicher macht und zu dessen Realisierung verhilft. Das Nummernschildbeispiel ist nur dessen kleinräumige Spielart; Fundamentaleres deutet sich in folgendem Votum Shelbys an:

\footnotetext{
"I have to believe in a world outside my own mind. I have to believe that my actions still have meaning, even if I can't remember them. I have to believe that when my eyes are closed, the world's still there. Do I believe the world's still there? Is it still out there? $[\ldots]$ Yeah. We all need mirrors to remind ourselves who we are. I'm no different."
}

Zum Problem stets interpretierter Fakten und dem sorgsamen oder sorglosen Umgang mit ihnen gesellt sich nun eine zusätzliche Herausforderung, jene nämlich, dass Erkenntnis und Interesse eine intransparente Liaison eingehen. Und Shelby verfügt über eminente Interessen.

Doch auch die "Fakten" und derjenige, für den sie »Fakten« sind, hinterliessen zusammen ein unvollständiges Bild; denn "Fakten« werden geteilt mit anderen; sie müssen sich in einem "Raum der Gründe und Begründungen« bewähren. Shelby ist für den korrupten Polizeispitzel Teddy und die drogendeal-affine Natalie ein gefundener Kandidat, um eigene Probleme loszuwerden, indem diese mit Shelbys Fall - entgegen der "Fakten" - verknüpft werden. Eine von den Tätern direkt eingestandene Manipulation des Protagonisten greift hier um sich, die seinen Status als Anti-Helden nur noch manifestiert.

Dies alles trägt nicht unbedingt dazu bei, die divergierenden Versionen zu den tatsächlichen Umständen von Shelbys Vergangenheit abwägen zu können: 
Version $A$ - Shelby hat Recht, d.h. im Wesentlichen ist alles so abgelaufen, wie er dem Unbekannten am Telefon schildert: Überfall / Mord nach Vergewaltigung an seiner Frau / er überlebt mit seinem »Zustand" / seine Geschichte ist die Suche nach dem zweiten Täter. - Nichts im Film widerspricht dieser Version in einer Weise, dass diese ausgeschlossen wäre. Der Film bietet also nicht negative "Fakten", sondern fächert bestenfalls ein Spektrum von Beweisen, Indizien, Vermutungen und Spekulationen auf, das nicht unberührt bleibt von derValidität der anderen Versionen.

Version B - Shelbys "Zustand « ist mit dem Fall von Sammy Jankis verbunden. "Verbunden" kann nun recht Unterschiedliches heissen. Filmischer Ausgangspunkt fürVersion $B$ ist eine flüchtige, leicht zu übersehende Szene, in der Sammy als Bebilderung Shelbys Erzählung von ihm in der psychiatrischen Klinik gezeigt wird; eine Person geht durchs Bild und darauf folgend ist anstatt Sammy für einen Moment Leonard im Rollstuhl sitzend $\mathrm{zu}$ sehen. Ist Sammys Geschichte (zumindest im Kern) die Leonards? Darauf deutet auch das, was chronologisch gesehen zu Beginn der Handlung durch Teddy eröffnet wird: Es gab den Überfall, doch Shelbys Frau überlebte und erlag erst später einem Insulinschock, nach dem sie ihren Ehemann auf die Wahrheit seines "Zustandes" testete, weil sie an der Wahrheit seiner Liebe für sie nie zweifelte. Leonard bestand den Test nicht; Version $A$ wäre nun die Verdrängung der Vergangenheit angesichts tragischer $>$ Schuld $<$ - in Anführungszeichen, da er den Insulintest gar nicht durchschauen konnte. Stimmten die Grundzüge derVersion $B$ nicht, hinge die besagte $>$ Rollstuhlszene in der Luft und es müsste zusätzlich ein Motiv ausfindig gemacht werden, warum Teddy die Unwahrheit gesagt haben sollte.

Version $C$ - Shelby ist eine Erfindung von Sammy Jankis: Im Grunde handelt es sich bei dieser Lesart um eine hardcore-Version, die zugegebenermassen in der Gefahr steht, davon zu leben, dass es gar kein festes Fundament gibt, welches etwas widerlegen oder bestätigen könnte. Dies ist nicht zuletzt deshalb zu beachten, da Memento kein Spiel vorführt, in dem durch traumhafte Bewusstseinssprünge alles erlaubt wäre. Es gibt auf der erzählten Ebene sehr wohl Regeln und Muster, die gegen eine um sich greifende Beliebigkeit sprechen. Und dennoch ist die Umkehrung derVersion $B$, in der Shelby seine Biographie mit dem Schicksal eines seiner Klienten verwebt, zu bedenken. Demnach würde nun ein Durchschnittspatient namens Sammy eine mit seinem eigenen Krankheitsbild ausgestattete Heldenfigur imaginieren, die genau das tut, was Sammy selbst unmöglich erscheint: zu kämpfen. 
Die Versionen $A$ bis $C$ umfassen weitere Derivate und sind für reichhaltigere Ausschmückungen offen. Doch wer an der Suche nach der treffenden Version interessiert ist, sollte zunächst die dazu vorgelagerte Frage beantworten, ob es in Memento wirklich darum geht, eine Entscheidung herbeizuführen. Die nun auszubuchstabierende These lautet, dass dieses Problem bestenfalls vordergründig bleibt - genauer: dass der Film vielmehr darauf angelegt ist, die relative Eindeutigkeit der Handlung zugunsten derVersion $B$ zusehends durch eine immer aufdringlichere Armada von Zweifeln zu ersetzen, die ihren Ort gerade nicht mehr in der Narration haben, sondern in deren Rezeption durch den Zuschauer: Nicht Shelby ist durchdrungen vom Zweifel trotz bester Gründe dafür, sondern wir als seine Begleitung kommen nicht umhin, uns dem Zweifel hinzugeben - in zweifacher Hinsicht ...

\section{VI.}

Die erste Hinsicht, in der der Zuschauer zum Zweifler wird, hat wiederum mit dem Anti-Skeptiker Shelby zu tun. Er erscheint als ein sympathischer Zeitgenosse, der diesen "Zustand « hat und somit orientierungslos, manchmal gar wie ein Kind wirkt, vor allem wenn er entschuldigend lacht und daher fast unser Mitleid verdient. Dieser Vertrauensvorschuss angesichts des Pathologischen färbt negativ auf die Nebenfiguren ab. Teddy ist ein kleiner, mässig korrupter Bulle, doch er gewinnt gerade in dem Moment an Glaubwürdigkeit, in dem er sein Vergehen, Leonard benutzt zu haben, zugibt. Jenseits dessen wollte er seinem vergesslichen Freund offenbar wirklich helfen, den zweiten Mörder zu schnappen. Ähnlich verhalten sich die Dinge bei Natalie. Zwar teilt sie nicht das frohe Gemüt des Polizisten John Gammell, da sie spröder und unzugänglicher wirkt, getrieben von Bedrohungen, die Leonard für sie lösen soll. Zuletzt jedoch unterstützt auch sie ihn in dosierter Aufrichtigkeit. Und nun greift der umgekehrte Mechanismus: Die vergleichsweise harmlose Kleinkriminalität der im doppelten Sinn supporting actors färbt negativ auf die Beurteilung Leonards ab; denn je deutlicher wird, dass erstere trotz moralischer Turbulenzen im Grunde gute Absichten hegen, tut der Film einiges dafür, Leonards Erscheinung einen dämonischen Zug zu verabreichen. Zweifel an seiner Integrität sind zunächst erlaubt, später unabweisbar.

Das einzige, was radikal böse genannt zu werden verdient, so Kant, ist der Wille. Indem Shelby den Dualismus von Interpretation und 
"Fakten" durchzuhalten versucht, "Fakten" aber nichts mit einem Willen zur Macht im Kleide der Interpretation zu tun haben, sondern ihm als gänzlich selbstevident erscheinen, gibt er vor, sich noch diesseits irgendeines Willens zu bewegen, nur geleitet von untrüglichen Tatsachen.

Diese zumeist lautlos mitlaufende Hintergrundannahme trifft hingegen nicht einmal Rudimente des Erzählten. Zunächst verdankt sich schon der Entschluss, nach dem zweiten Mann zu fahnden, einem Willen, der kaum selbstverständlich zu nennen ist. Die durchschnittliche moralische Empörung über einen Mann mit dem "Zustand", der über Leben und Tod von mehreren John G.s entscheidet, würde auf Verantwortungslosigkeit plädieren. In der Tat ist Leonards Plan wahnsinnig, zum Schluss hybrid. Doch Entscheidendes kommt hinzu: Gemäss Version $B$ ist gegen Ende des Films (d.h. gleich zu Beginn der chronologischen Handlung) deutlich, dass Shelbys Rachephantasien das perfide Resultat eines psychologisch zumindest erklärlichen Selbstbetruges sind. Nolans inszenatorischer Gestus der Umkehrung der Erzählrichtung fungiert hier als Verschleierung dieses zuletzt simplen Zusammenhangs, wodurch die schrittweise Unterminierung der Hauptfigur an Drastik gewinnt und mit ihr die Momente des Zweifels derer, die ihn zuschauend begleiten, prominenter werden. Die Manipulation der Daten (vgl. nochmals die 'Nummernschildaktion ), die zunehmende Selektion von Informationen (aufschreiben oder vergessen?), sein eigenes Ver- oder Misstrauen anderen gegenüber, das die geleugnete Interpretation am Faktischen schon immer mit sich führt (besonders bei widersprüchlichen Kommentaren auf seinen Polaroids) - all diese Untiefen des Externen sind letztlich nur Implikate einer Selbstmanipulation.

Auf beiden Ebenen, der erzählten und der erzählenden, ist Shelbys Amnesie als alles entschuldigendes Moment auch des Äussersten, des Mordes an Unbescholtenen, etabliert. Der Zweifel des Zuschauers führt in Umwegen dazu, diese Entschuldigung nicht länger gelten zu lassen. Shelby will durchaus etwas, er will töten, wie am Mord an seinem Freund Teddy gleich zu Beginn des Films gezeigt wird. Wie fatal falsch das gewesen ist, zeigt die anachrone Fortsetzung, wovon unsere Einstellung Leonards gegenüber zunehmend bestimmt wird. Die Indizien dafür, dass John Edward Gammell der gesuchte (und schon längst gefundene und in Person von Jimmy Grants >noch einmak gefundene) John G. sei, waren nichts als lächerlich. Ein falsches Kennzeichen und der gebräuchlichste christian name im Englischen samt nichts sagendem Initial des Familiennamens waren die »Fakten«, 
aus denen Shelby übersprungshaft tödliche Schlussfolgerungen gezogen hat.

Der gute Leonard durchschaut sehr wohl die Logik der Rache, in deren Dienst er seiner verdrehten Vergangenheitsversion zufolge steht. Und daher fragt er nach dem Sinn von Rache für seine misshandelte und getötete Frau, wenn er doch die einmal vollzogene Rache sogleich wieder vergisst. Auch hier gelangt Shelby zu nicht mehr als labilen Eindeutigkeiten:

Natalie: "But even if you get revenge you're not gonna remember it.You're not even going to know that it happened."

Shelby: "My wife deserves vengance. Doesn't make a difference whether I know about it. Just because there are things I don't remember doesn't make my actions meaningless. The world doesn't just disappear when you close your eyes, does it? Anyway, maybe I'll take a photograph to remind myself, get another freaky tattoo."

Shelby vertritt hier einen Realismus der Rache, ohne den sein Unternehmen entweder unmöglich oder zumindest unverhältnismässig erschiene. Unmöglich, insofern Rache immer gefühlte Rache ist, die ein (partielles) Erinnern voraussetzt - ähnlich wie Schmerzen, die nicht gespürt werden, keine Schmerzen sind, und Leiden, das dem Opfer nicht als solches vorkommt, kein Leiden ist. Unverhältnismässig, insofern die gefühlte Rache nicht anhält und die Genugtuung in keiner Relation zu dem stehen mag, worauf sie antwortet, weshalb neue Objekte vollzogener Rache erforderlich werden.

Der in diesem Sinn realistische Shelby würde beides abweisen, doch der Film widerspricht auch hier seiner Hauptfigur. So wird ein von Teddy geschossenes Foto gezeigt, auf dem Leonard glücklichlächelnd nach dem Mord an dem zweiten Mann zu sehen ist. Sein Gesicht steht in Kontrast zu seiner realistischen These. Will er uns wirklich weismachen, dieses Gefühl sei ganz unverbunden mit dem Akt der Vergeltung? Auch hier scheint der gute/böse Leonard einer Konfusion aufzusitzen, zu der sich ein letztes, ihn in seinem >Wesen überführendes Moment gesellt: Leonard wusste nach dem ersten Mord, dass er John G. gefunden hatte. Warum hat er sich nicht ein Foto von der Leiche gemacht, die ihn daran unwiederruflich erinnert, dass er seine vermeintliche Mission längst erfüllt hat? 
Er hatte damals die Wahl, es festzuhalten und ist dem Imperativ memento nicht gefolgt - mit Kant: willentlich! Ist er also böse?Vielleicht wären wir es, wenn wir ihn nicht dafür halten würden.

\section{VII.}

Die zweite Hinsicht unseres zuschauenden Zweifels hat nun damit zu tun, dass uns Memento nicht im Modus eines passiven Publikums verharren lässt, sondern uns durch sein Anordnungsprinzip der Szenen mit zunehmend spürbarer Notwendigkeit in diesen Kriminalfall involviert. Die anachrone Abfolge der für sich recht überschaubaren Passagen gewinnt an Unübersichtlichkeit durch Nolans Spiel mit unseren temporalen Gepflogenheiten. Dabei ist der Film durchaus linear strukturiert, aber eben in konsequenter Drehung. Der skizzierte Rückgang ins zeitlich Vorangehende bricht mit unseren rezeptiven Routinen von kausalen Folgen zwischen Absichten und Effekten. Der erzählerische Endpunkt, der Mord an Teddy, eröffnet den Film, wodurch nicht mehr die Lösung einer filmisch vorbereiteten Herausforderung im Mittelpunkt steht, sondern die der Lösung vorgelagerte Präparierung.

Version B zufolge geht die Sache ziemlich schlecht aus: Leonards Recherchen ergeben allein für ihn diejenige Konsequenz, die er in selbst manipulierten Indizien ziehen zu müssen meinte: Teddy sei der gesuchte John G. Am Ende von Memento weiss der Zuschauer, dass Leonard in gleich mehrfachem Sinn völlig irrte, was den immer vehementeren Eindruck verstärkt, als Zuschauer der Tugend nachkommen zu sollen, die der Protagonist permanent mit Füssen tritt: Umsicht. Was Shelby vollkommen abgeht, ist - um es technischblumig zu wenden - epistemische Demut; sein "Zustand" dient ihm nicht nur als Herausforderung für umfängliche Ermittlungen, sondern gleichzeitig als unausgesprochene Entschuldigung des mörderisch Äussersten. Das Gefühl dafür, in welcher prekären Lage der als Ermittler hemdsärmliche Leonard steckt, überträgt sich durch Nolans anachrone Erzählweise auf den Zuschauer: Seine Orientierungslosigkeit wird unsere; der Film macht unaufdringlich mit uns, was er an Shelby so elegant zeigt. Wir werden zu Detektiven, um dem Film folgen zu können.

In diesem Sinn darf Memento performativ genannt werden, weil sich Leonards Amnesie in die rezeptive Erfahrung des Zuschauers einschleicht: Wann begreift man, dass umgekehrt erzählt wird? Wie lange vermag man, halbwegs souverän die Handlung wieder um- 
zudrehen, ohne selbst etwas zu verdrehen? Wie schnell gelingt es dem Film, einem die Orientierung zu nehmen, sobald man bemerkt, dass prinzipielle Zweifel an allen und allem angebracht sind? Gerade weil Skepsis für das ganze Figurenkabinett ein Fremdwort darstellt, ist der Verdacht in uns gesät: Wir übernehmen folglich, was vor allem Leonard gut getan hätte. Darum ist Mementos Erzählweise kein Selbstzweck, sondern konstituiert die Möglichkeit zu erfahren, was als erzählte Erfahrung nur behauptet werden kann.

Auch die Überschaubarkeit des Falles, den Leonard zu lösen vorgibt, mit dem er aber schon überfordert zu sein scheint, spiegelt sich in dem Fall, den wir übernehmen, indem wir Memento sehen. Keineswegs wird eine extravagant verwinkelte Geschichte erzählt; das Gewöhnliche reicht völlig aus, um in uns Verwirrung zu stiften. Diese Stiftung verdankt sich auch nicht unlauterer Mittel, etwa dem oft vermuteten Zug, die Rückführung der anachronen Szenen ergäbe keine lückenlos lineare Geschichte. Das trifft nicht zu; Memento geht 'fair mit dem Zuschauer um und kann sich für die Evidenz seines Effekts eben gerade auf diese Stringenz berufen. So zeitig also setzt die Amnesie auch bei uns ein! Keine szenischen Überwältigungen, nicht einmal verschleiernde Ablenkungen, lediglich die Umkehrung der Szenenfolge.

Das Resultat ist so schlicht wie einleuchtend: Wir geraten in Shelbys Situation, für deren Handhabung der Film zugleich unausgesprochene, aber bebilderte Vorschläge bieten kann. Wir sind nun stets gefordert, uns die Sequenzen einzuprägen - wie Shelby, der Fotos gegen das unmittelbare Vergessen schiesst; wir sind versucht, wie in Kinderjahren Dinge auf die Hand zu schreiben - wie Shelby, der sich regelmässig in Tattoostudios begibt; wir sind dabei, innere Notizen anzulegen, um weiter folgen zu können - wie Shelby, der Kurzkommentare an den Rand der Polaroids verfasst. Irgendwie muss der Rasanz des Vergessens entgegengearbeitet werden. Durch diesen Transfer vom Gezeigten zum Getanen, vom Schicksal der Hauptfigur bar jeder Skepsis zum zweifelnden Engagement unserer selbst geht Memento zuletzt über das rein Performative entscheidend hinaus, indem er an uns tut, was er an Shelby zeigt. Der Film wandelt sich zum Sakrament.

- Dr. Hartmut von Sass ist wissenschaftlicher Assistent am Lehrstuhl für Systematische Theologie, Symbolik und Religionsphilosophie der Universität Zürich und Associate Fellow am Collegium Helveticum. 